\title{
Design of Micro-Controller Based Automatic Electrical N/S source switcher Between 3 Electrical Sources: University Hassan the first Morocco Data Center's energy effectiveness use development
}

\author{
Zakarya Benizza, Ahmed Mouhsen, Anis Kirama
}

\begin{abstract}
The automatic electrical N/S sources switcher, is build using a PIC16F877A micro-controller and electrical input/output sensors. This system is programmed in $\mathrm{C}$ language using the MikroC compiler. It's accurate, smart, flexible and easily configurable. It is designed to automatically get instantaneous measurements of the currents; Voltages delivered by three sources of energy, also the levels of photovoltaic batteries, the level of diesel and, also temperature inside and outside of the server room. And to deduct the consummate power and the power factor from it. And to switch towards the suitable source of energy according to the parameters configured in its program.

The measurements are collected with repetitions rate tuneable from one to sixty minutes, are archived in an application especially developed for this system, and displayed on a LCD central monitor. The processing of these information's relating to the instructions configured by the operator, allowed to connect with efficiency the Data center, on one of the three source of electrical energy: Normal source ONE (Moroccan electricity provider), first Emergency photo-voltaic source, and the generating diesel source.
\end{abstract}

Index Terms - Micro controller, electrical sources switcher, Sensors, C language, energy effectiveness, Data-center.

\section{INTRODUCTION}

Data center is the most critical element on the base of any information system IS, it accommodates all the servers, routers, switches, fire-wall and the other computing equipment which have for role to treat, protect and secure, so to insure the access to the information, and which consist since all the times a basis in the putting of the strategies process and the decision-making.

In front of the vulgar growth of the volume of the information handle in their Data center, many of companies -universities choose to externalize the IT service to other Companies expert in this kind of service and which use generally the techniques of cloud and virtualization.

This convergence is to lash by the budget dedicated to the conception of IS, and the criticality of the information to the databases and servers.

Zakarya BENIZZA, Departement of Technologies, Hassan $1^{\text {st }}$ University, Faculty of Sciences and Technologies FST, Settat, Morocco +212661887310 (z.benizza@uhp.ac.ma).

Ahmed MOUHSEN, Departement of Technologies, Hassan $1^{\text {st }}$ University, Faculty of Sciences and Technologies FST, Settat, Morocco +212672125214 (mouhsen_ahmed@yahoo.fr).

Anis KIRAMA, Departement of Technologies, Hassan $1^{\text {st }}$ University, Faculty of Sciences and Technologies FST, Settat, Morocco +212614270163 (a.kirama@uhp.ac.ma).
Further to pieces of the new reforms, which encourage the integration of information technologies and communication TICE, in the education process of Moroccan universities, they are not any more seen from the same angle, the Moroccan university today seen by the eyes of students and professors is a real provider of IT services (E-learning, MOOC, E-mail, WIFI, Web, Radio...). For the decision-makers, it is a well of information and statistics managed by the ERP and that direct the decision-making.

In these conditions, and to answer to their user's needs, a Data center (IT equipment / Facility) [5] became an essential component at the heart of the Moroccan digital university.

The Hassan 1st Settat-Morocco university, - which is the case of our studies, is Among Moroccan universities who anticipated this change, the Data center exists since 2010, but it always classified in the rank of underperforming Data centers in the maturity model elaborated by the Green Grid [5].

The objective of our study, is the improvement of the energy efficiency of the university's Data center to climb on the scale of ranking, by acting on the PUE (power usage effectiveness) of the server room, through the management of the power supplies by a concept of a device named automatic electrical N/S source Switcher which manage and automates the electrical feed of data center through 4 functioning modes.

Nowadays, new electronic components and integrated circuits produced by various manufacturers and dedicated to the electronic instrumentation are available in the market. These are, for example, removable memories such as flash disks capable to store a large amount of data and programmable logic devices like microcontroller, DSP (Digital Signal Processor) and FPGA (Field Programmable Gate Array), used to build data loggers and automatic measurement systems. Moreover, small sensors today commercialized, are able to measure different phenomena and convert them into electric signals; the automatic electrical N/S sources switcher is built using the PIC 16F877A microcontroller. Such a microcontroller has been chosen owing to its very good performance, programming facilities, availability and economical advantages.

\section{DESIGN OF THE AUTOMATIC ELECTRICAL N/S SOURCES SWITCHER}

The block diagram of Figure 1 shows that the automatic electrical N/S sources switcher's system under study mainly is based on three electric sensors respectively measuring currents delivered by 3 electrical sources, temperature inside 
and outside the server room, and gasoline level their matching circuits, a PIC 16F8877A microcontroller, an LCD (Liquid-Crystal Display) used as alpha- numerical display, and an RS232 serial interface.

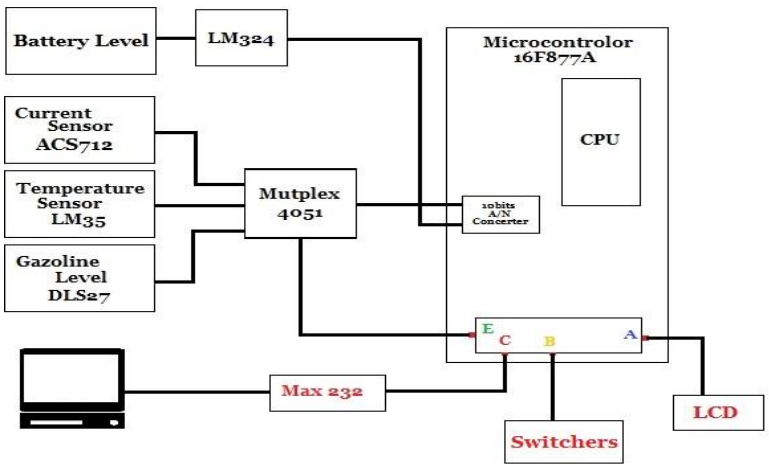

Figure 1. Block diagram of the meteorological data acquisition system.

The microcontroller insures the management of the whole management system of power supplies.

It's receives the resulting signals delivered by the sensors, it handles the immediate states information of the tree power supplies: values of the temperatures, quantity of load stored in photovoltaic batteries, level of diesel oil in the reservoir of the generator, display of these sizes on a LCD monitor, so transfer of these data via an interface RS232 towards a database on which they are stored and to consult afterward via an Visual basic application developed especially to present the indicators of the system.

The PIC 16F877A is as efficient as the other programmable logic devices and much cheaper than FPGA, DSP or most of the other microcontrollers.

\section{SENSORS MEASUREMENTS}

\section{A. Currents Measurement}

The currents of the three electrical sources, are measured Figure 2 using the ACS712 sensor of Allegro. The device consists of a precise, low-offset, linear Hall circuit with a copper conduction path located near the surface of the die. Applied current flowing through this copper conduction path generates a magnetic field which the Hall IC converts into a proportional voltage. Device accuracy is optimized through the close proximity of the magnetic signal to the Hall transducer. A precise, proportional voltage is provided by the low-offset, chopper-stabilized BiCMOS Hall IC, which is programmed for accuracy after packaging.

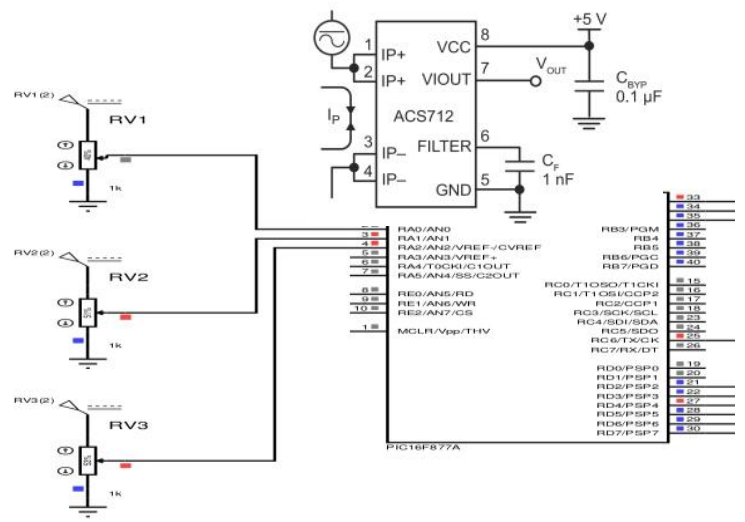

Figure 2 : Current sensor and it's matching Circuit
The output of the device has a positive slope (>VIOUT(Q)) when an increasing current flows through the primary copper conduction path (from pins 1 and 2, to pins 3 and 4), which is the path used for current sampling. The internal resistance of this conductive path is $1.2 \mathrm{~m} \Omega$ typical, providing low power loss [1].

The sensor ACS712 gives a precise measure of the current for both signals AC and DC. Thick copper driver and tracks of signal allows the survival of the device until 5 times the conditions of overload. THE ACS712 emits an analogue output signal of tension, which varies linearly with detected current. The device requires $5 \mathrm{~V}$ DC and a couple of condensers of filtering. It has a sensibility of $185 \mathrm{mv} / \mathrm{A}$.

\section{B. Temperatures Measurement}

The temperature is measured Figure 3 with the LM35 sensor, this small circuit of TEXAS INSTRUMENT, has three pins to measure a temperature of $-55^{\circ} \mathrm{C}$ to $+150^{\circ} \mathrm{C}$. this sensor sensitivity as $10 \mathrm{mV} /{ }^{\circ} \mathrm{C}$ which gives $250 \mathrm{mV}$ at $25^{\circ} \mathrm{C}$. The LM35 series are precision integrated-circuit temperature devices with an output voltage linearly- proportional to the Centigrade temperature.

The LM35 device has an advantage over linear temperature sensors calibrated in Kelvin, as the user is not required to subtract a large constant voltage from the output to obtain convenient Centigrade scaling
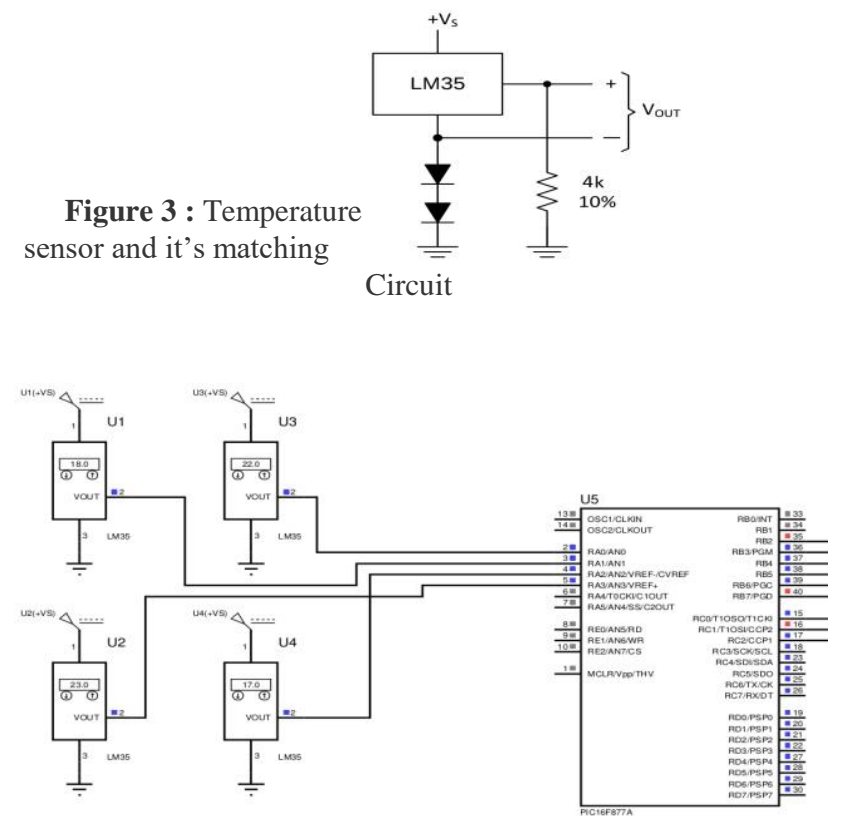

The LM35 device does not require any external calibration or trimming to provide typical accuracies of $\pm 1 / 4^{\circ} \mathrm{C}$ at room temperature and $\pm 3 / 4^{\circ} \mathrm{C}$ over a full $-55^{\circ} \mathrm{C}$ to $150^{\circ} \mathrm{C}$ temperature range. Lower cost is assured by trimming and calibration at the wafer level. The low-output impedance, linear output, and precise inherent calibration of the LM35 device makes interfacing to readout or control circuitry especially easy. The device is used with single power supplies, or with plus and minus supplies. As the LM35 device draws only $60 \mu \mathrm{A}$ from the supply, it has very low self-heating of less than $0.1^{\circ} \mathrm{C}$ in still air. The LM35 device is rated to operate over a $-55^{\circ} \mathrm{C}$ to $150^{\circ} \mathrm{C}$ temperature range, while the $\mathrm{LM} 35 \mathrm{C}$ device is rated for a $-40^{\circ} \mathrm{C}$ to $110^{\circ} \mathrm{C}$ range $\left(-10^{\circ}\right.$ with improved accuracy) [2]. 


\section{Gasoline Level Measurement}

In our system we need is to maintain a minimum level of fuel in the tank for use for the generator. Level detection Figure 4 is done using The DLS-27N-30. The level sensor DLS-27 has no moving parts, it works on capacitive principle.
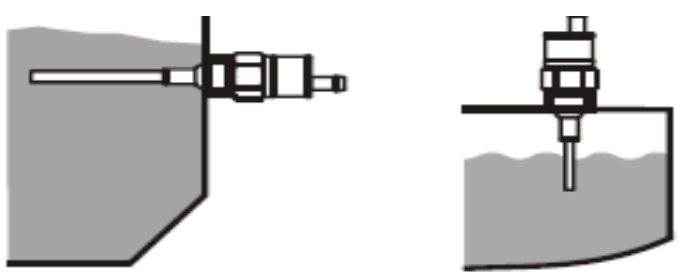

Figure 4 : Gasoline Level sensor

Its electrode (rod, rope, etc.) makes the capacitor together with the metallic housing (and consequently the metallic walls of a vessel or other near objects). The capacity of the capacitor is done by the dimensions of the electrode and the dielectric properties of the surrounding medium (its permittivity). When the change of the permittivity occurs - by fully or partly immersion into the matter - the capacity changes its value. This change is detected by electronics inside the DLS and makes the state change on its output [3].

\section{Batteries Level Measurement}

The battery level is measured Figure 5 by monitoring the voltage supplied by each of the batteries assembled in series, this measurement will be provided by the Microcontroller through a voltage adaptation module by using a step-down voltage amplifier stage which allows divide the input voltage by 2.4 to deliver $5 \mathrm{v}$ at the output when $12 \mathrm{v}$ delivered by the battery. The LM324 amplifier of Texas Instruments provides the amplification stage.

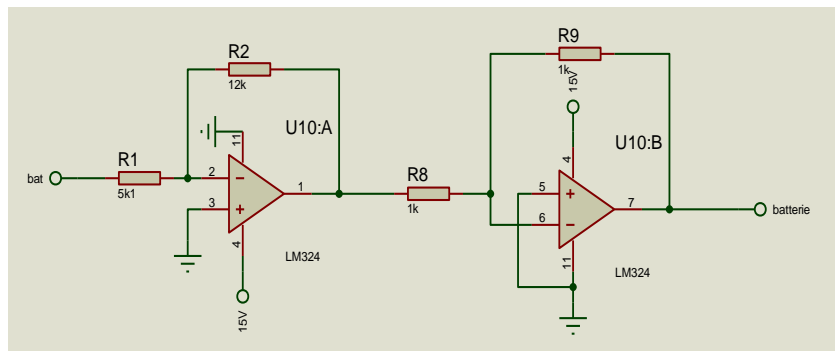

Figure 5 : Batteries level Amplifier and it's matching Circuit

The LM324 series are low-cost, quad operational amplifiers with true differential inputs. They have several distinct advantages over standard operational amplifier types in single supply applications. The quad amplifier can operate at supply voltages as low as $3.0 \mathrm{~V}$ or as high as $32 \mathrm{~V}$ with quiescent currents about one-fifth of those associated with the MC1741 (on a per amplifier basis). The common mode input range includes the negative supply, thereby eliminating the necessity for external biasing components in many applications. The output voltage range also includes the negative power supply voltage [4].

\section{BUILDING AND PROGRAMMING THE MiCROCONTROLLER CARD}

PIC 16F877A is a microcontroller manufactured by Microchip following the CMOS technology. Supplied with only $5 \mathrm{~V} \mathrm{dc}$, it consumes very little electrical energy. The number of its pins equals 40 , including 33 in- puts/outputs. It works at $20 \mathrm{MHz}$ provided that $20 \mathrm{MHz}$ quartz is connected at the pins of its internal oscillator. The internal structure of PIC 16F877A integrates all the components of a computer. It is mainly composed of its ALU (Arithmetic and Logic Unit), internal clock, three timers, five input/output ports, Analogue-to-Digital converter having eight 10-bit channels, RAM (Random Access Memory), EEPROM (Electrically Erasable Programmable Read-Only Memory), Flash memory and interface circuits such as USART (Universal Synchronous Asynchronous Receiver Transmitter), MSSP (Master Synchronous Serial Port) and PSP (Parallel Slave Port) [6] .

In our system the A Port is used to measure current (Normal source, Emergency source 1, Emergency source 2), the level of fuel, the internal and external temperature (inside and outside the server's room). These phenomena are measured through an analogue multiplexer 4051 which is an integrated circuit made by TEXAS INSTRUMENT, with 16 pins that has as role to bring out the 8 input by the single output based on statements of $\mathrm{ABC}$ selections bits. Also measuring the battery's level with a level matching circuit based on the amplifiers LM324, this arrangement ensures a voltage division by 2.4 to achieve $5 \mathrm{v}$ when the entry voltage is equal to12v.

The E port of the PIC16F877A is used for 3-bit selection multiplexer; the B Port is used to send control signals to the switchers, which control with a harmonious way the whole system. The C Port is used for communication with the computer using the UART module through the Max232 module that converts TTL levels $(05 \mathrm{v})$ to RS232 level $(+12 \mathrm{v}$ $-12 v)$.

The D Port is used to display the results on an alphanumeric LCD display. The PIC16F877A is configured using the MikroC for PIC compiler developed by the $\mathrm{C}$ language, PICKIT2.

The microcontroller is programmed by following these steps:

i. Write the program to the functioning of our system using the MikroC for PIC compiler in C language.

ii. After compiling the program we get a hexadecimal file (.HEX).

iii. We place our PIC programmer on PICKIT2.

iv. We open the software on PICKIT2 transferring the generated .HEX file.

v. PICKIT2 programmer is connected to a PC via the USB bus.

vi. Le programme .HEX is transferred to the PIC.

\section{THE SYSTEM's 3D STRUCTURE}

The automatic switch system N/s is represented under its 3D structure; allowing to visualize various component of the system, and which are decomposed into two categories: 


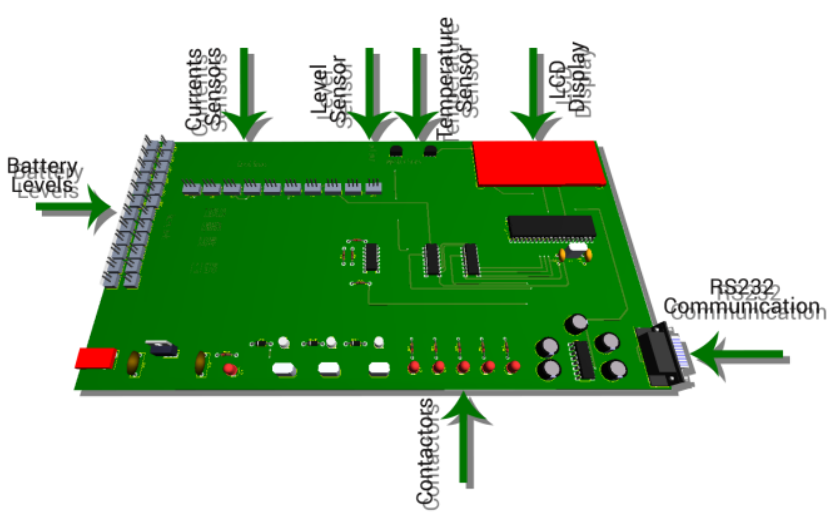

Figure 6 : The automatic electrical N/S sources switcher 3D Structure

i. System input Entries

- The signals coming from the current's sensors of the three-power supply.

- The signals coming from the batteries level's Sensors of the second source of energy (solar).

- The signal coming from gasoline level's Sensor in the reservoir of the generator, which constitutes the third electric source of energy.

- The signals coming from the temperature's Sensors inside and the outside of the server room.

ii. Heart of treatment in the system

- The PIC16F877A

- Arithmetical and logical processing units.

- Flash/RAM memory.

\section{iii. System output Entries}

- The states of the switcher up/down indicated by a LED Green/Red.

- The connector towards the LCD screen.

- The RS232 port, allowing the communication with the central computer, to display and store information about the entries.

\section{TEST AND MEASUREMENTS:}

To test the functions of our system we are going to represent it as following in the figure in 2 categories:

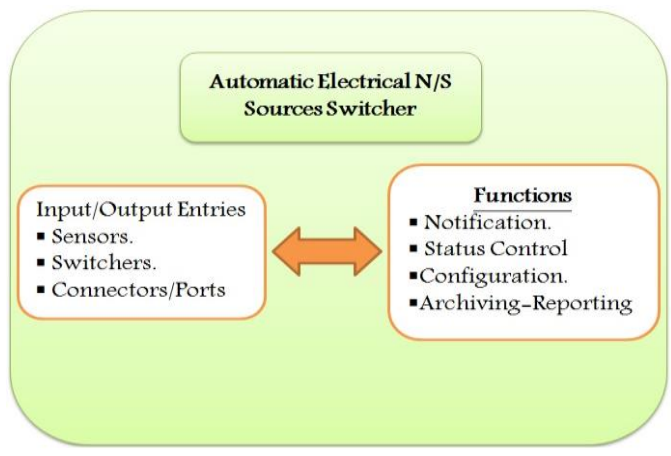

Figure 7: Automatic switcher functions

\section{i. Notification function}

The system allows the real time notification of the states of the power supply (ONE source, Emergency solar source, Emergency Generator source) SN, ES1, and ES2 and also the states of the switchers Qn, QC1, QC2, QT, Poff.

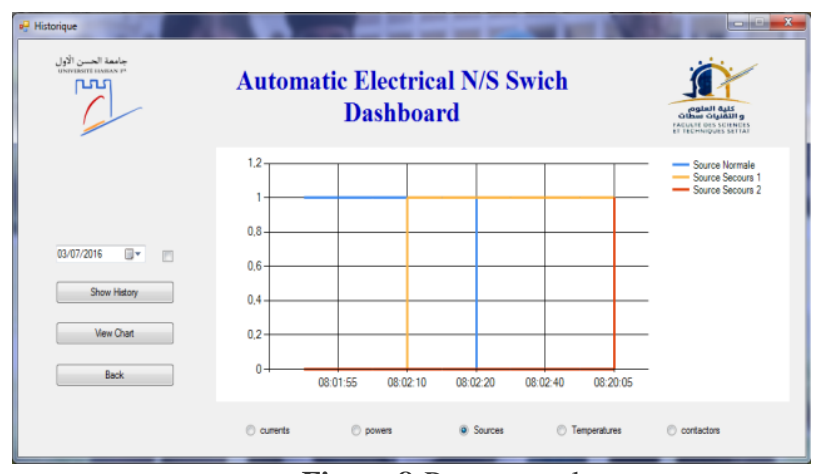

Figure 8:Power supply

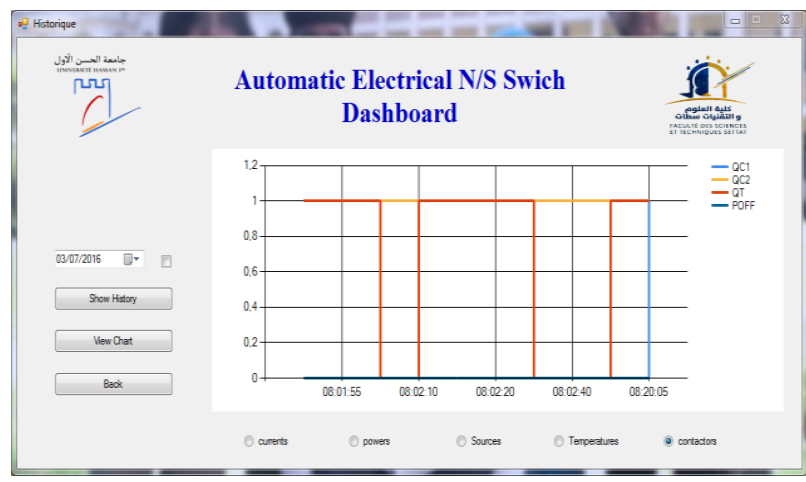

Figure 9: Switchers states

ii. Status Control function

The system figure 8 allows to check, measure and calculate in real time the physical quantities IN1, IN2, IN3, IS11, IS12, IS13, IS21, IS22, IS23, PN, CosQ, PS1, PS2, Ti, Te, NG, NB. This status are visualized by a virtual basic application developed for the system as shown in the figure this entries can take various forms Up/Down represented by LED green/Red, also values were spotted by digital character or percentage.

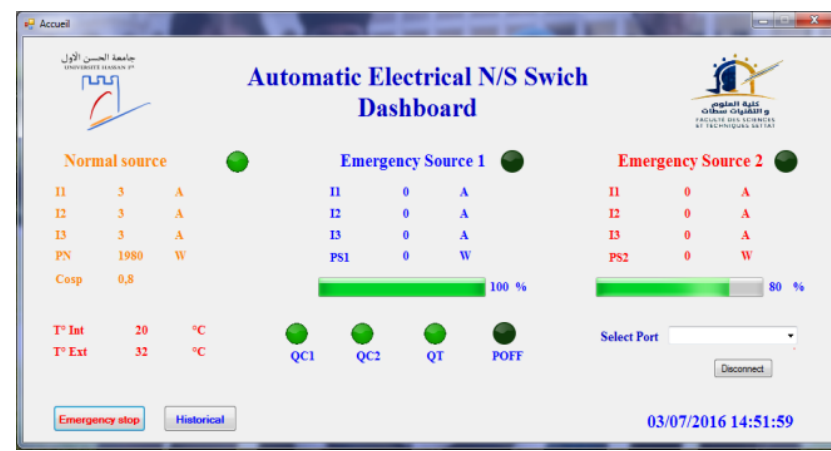

Figure 10: Digital Values/status of the input/output Entries

iii. Configuration function

The system allows to modify the configuration and automation of the instruction instrumenting the functioning. Through line scripts its program acts and reacts according to state of input/output entries to insure the 4 mode of functioning (Normal Mode, Emergency1 mode, Emergency2 mode, Shutdown mode).

\section{iv. Archiving-Reporting function}

The reporting figure 9 in this kind of system remains an essential function, in this theme; we conceived an intelligent system, which insures the archiving input/output data in 
MysqlServer databases, which monitored by the PhPMyadmin solution.

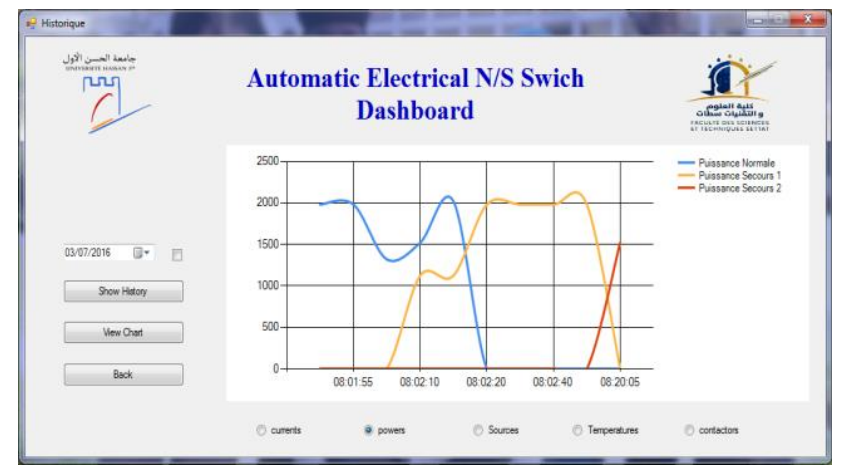

Figure 11: Powers delivered reports

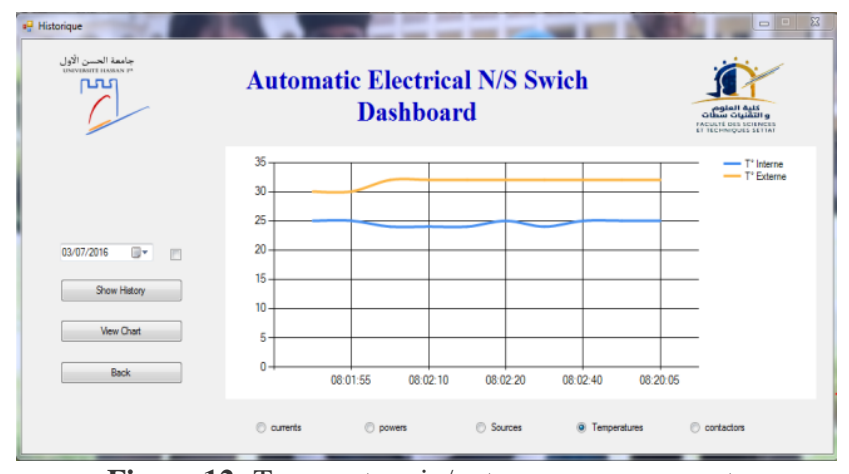

Figure 12: Temperature in/out server room reports

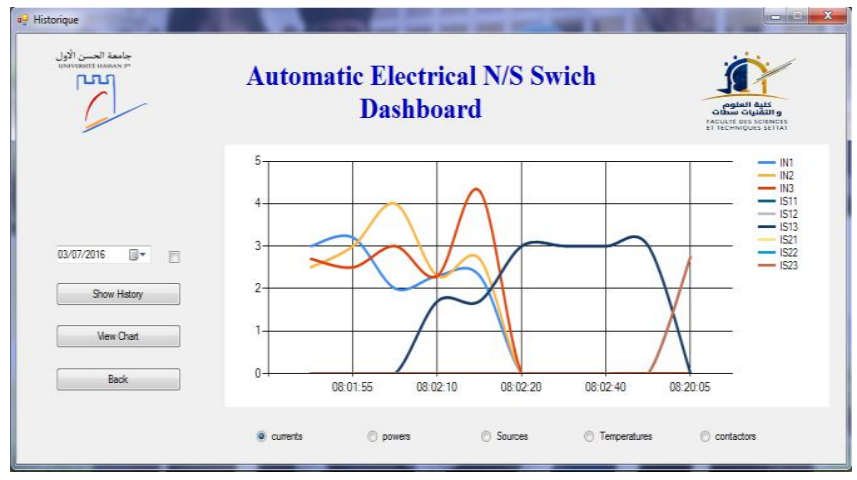

Figure 13: Currents delivered reports

The person in charge of the Data center can easily viewed these informations and generate various kind of reports: snapshot state, daily, monthly, yearly reports).

\section{CONCLUSIONS}

During the testing and simulation phase conducted at Hassan 1st University Settat, the automatic electrical N/S sources switcher gave fascinating results about the energy consumption of the data center. This is due to the quality robustness of the sensors and the micro-controller used to measure and treat physical quantities (currents, temperatures, battery, fuel level....).

The following data are displayed on an LCD screen and transferred in real time via the RS232 connector to a central computer, where they are viewed and treated in the archive target to derive detailed status reports.

The instrumentation of electric functioning, make the electrical feed operations more efficient and redundant. Also the processing of this data allows the Data center responsible to visually and automatically manage the Data Center's power supplies changes

During the design of the automatic electrical N/S sources switcher, we have taken into consideration the homogeneity, flexibility and adaptability of the system to the Data Center in the Moroccan university context and in a specific way the context of the University Hassan 1st Settat.

Sure the work does not stop there, the team is studying the possibility of developing a second version of the system, which can calculate in real time the powers consumed in separate block (Facility / It Equipment) and deduce the PUE energy efficiency factor.

\section{ACKNOWLEDGEMENTS}

Many thanks to Dr. FAHLI Ahmed the vice president in charge of scientific research in Hassan 1st University Settat, and his team for the total access to the Data Center and the other building in relation with, which helped us in our studies, Tests and simulations.

\section{REFERENCES}

[1] 1] ACS712-Datasheet (16 nov. 2012) - 1 nF. Application 1. The ACS712 outputs an analog signal. Allegro Microsystems http://www.allegromicro.com/ /media/Files/.../ACS712-Datasheet.as hx

[2] [2] LM35 Precision Centigrade Temperature Sensors (5 janv. 2016) and precise inherent calibration of the LM35 device Texas Instruments http://www.ti.com/lit/ds/symlink/lm35.pdf

[3] [3] Capacitive level sensors DLS-27 (07. 2007) Dinel, Ltd., U Tescomy 249, CZ $\quad-\quad 760 \quad 01 \quad$ Zlín http://www.lectrotek.com/datasheets/agency.products/DLS-27-dat-en _DS.pdf

[4] [4] LM2902-N, LM324-N. SNOSC16D -MARCH (2000)-REVISED JANUARY $2015 \quad$ LMx24-N, LM2902-NLow-Power, Quad-Operational Amplifiers Texas Instruments http://www.ti.com/lit/ds/symlink/lm124-n.pdf

[5] [5] Data Center Maturity. Model (DCMM) (2012) Green Grid White Paper http://www.thegreengrid.org/ /media/WhitePapers/Data\%20Center\% 20Maturity\%20Model\%20White\%20Paper_final.ashx?lang=en

[6] [6] Amar Adane, Ania Ali Yahia, Essaid Mameri, Abd-El-Hamid Adane, (28 August 2014) Design of a Microcontroller-Based Data Acquisition System for Ground Weather Observations: Evaluation of Radio Refractivity of Air Scientific Research Publishing Inc. http://www.scirp.org/journal/PaperInformation.aspx?PaperID=49420

[7] [7] Crisp, J. (2004) An Introduction to Microprocessors and Microcontrollers. Elsevier, Boston.

[8] [8] Bolton, W. (2009) Programmable Logic Controller. 5th Edition, Newnes, Oxford.

[9] [9] Hartmut, F., Sadrozinski, W. and Wu, J.Y. (2010) Applications of Field-Programmable Gate Arrays in Scientific Re- search. Taylor \& Francis, Boca Raton.

[10] [10] Asch, G. (2010) Les capteurs en instrumentation industrielle. 7th Edition, Dunod, Paris.

[11] [11] Özcan, Ö. and Gündüz, M. (2014) Investigation and Implementation of a PIC-Based Sensor Node for Wireless Sensor Networks. Journal of Computer and Communications, 2, 90-98. http://dx.doi.org/10.4236/jcc.2014.24013

[12] [12] Bhaskar, P., Bhagyajyoti and Immanuel, J. (2012) Design and Development of Microcontroller-Based Photoacoustic Spectrometer. Sensors \& Transducers, 14, 26-34.

[13] [13] Tavernier, C. (2005) Programmation en C des PIC. Dunod, Paris 